\title{
A necessidade de fortalecer os cuidados paliativos na saúde pública do Paraná
}

\author{
The need to strengthen palliative care in the public \\ health in the State of Paraná
}

Fernando Cesar Iwamoto Marcucci ${ }^{1}$, Luís Fernando Rodrigues ${ }^{2}$, Marcio José de Almeida $^{3}$

\begin{abstract}
1. Fisioterapeuta. Doutor em Saúde Coletiva. Hospital Dr. Anísio Figueiredo - Zona Norte de Londrina. Secretaria de Saúde do Paraná. Email: fcim@msn.com. ORCID: http://orcid.org/0000-0001-8139-7772

2. Médico. Mestre em Cuidados Paliativos. Hospital São Judas Tadeu - Hospital do Amor/Barretos. Email: lufe.luis@gmail.com. ORCID: http://orcid.org/0000-0001-6177-3769

3. Médico. Doutor em Saúde Pública.Professor da Faculdades Pequeno Príncipe. Email:marciojalmeida2015@ gmail.com. ORCID: http://orcid.org/0000-0001-7094-9906

CONTATO: Fernando C. I. Marcucci | Rua Henrique dos Santos, 62 - Apto. 701 | CEP 86015-150 | Londrina | Paraná | Telefone: (43) 99912-3929 | E-mail: fcim@msn.com
\end{abstract}

COMO CITAR: Marcucci FCl, Rodrigues LF, Almeida MJ. A necessidade de fortalecer os cuidados paliativos na saúde pública do Paraná. R. Saúde Públ. Paraná. 2020 Jul;3(1):18-30.

RESUMO Este estudo busca identificar a demanda dos cuidados paliativos (CP) no estado do Paraná, refletir sobre a legislação, fundamentos e prática desta abordagem, com propostas para sua inserção no SUS. Analisou-se a demanda de CP no Paraná, por meio da análise da mortalidade, revisão da legislação relacionada ao tema e apresenta reflexões e propostas para a inserção dos CP nas Redes de Atenção à Saúde (RAS). Cerca de 70\% das mortes no estado, em 2018, ocorreu na faixa etária acima de 60 anos, 63,8\% destas tinham potencial necessidade de CP no fim de vida, $66,6 \%$ ocorreram nos hospitais e $21,3 \%$ no domicílio, com variações entre as regionais de saúde. Houve avanços recentes na legislação sobre a inserção de CP no sistema de saúde, mas ainda há necessidade de definir e ampliar as ações para o maior acesso à esta forma de abordagem, nos diversos níveis de atenção à saúde. 
PAlAvRAS-Chave: Cuidados Paliativos. Política de Saúde. Serviços de Saúde. Saúde Pública. Sistema Único de Saúde.

\begin{abstract}
This essay aims to identify the demand for palliative care (PC) in Paraná State (Brazil), to discuss the legislation, fundamentals and practice of this approach, with proposals for its insertion in SUS. The demand for PC in Paraná was analyzed through public database of mortality; related legislation was reviewed and it presents considerations and proposals for the insertion of PC in Healthcare Networks. About $70 \%$ of all deaths in the state, in 2018, occurred in the age above 60 years old, $63.8 \%$ of them had a potential need for PC at the end of life stage, and $66.6 \%$ occurred in hospitals and $21.3 \%$ at home, with variations among state's health regionals. There was recent advances in legislation on the insertion of PC in the healthcare system, but there is a need to define and expand actions for greater access to this kind of approach, at all levels of health care.
\end{abstract}

KEYWORDS: Palliative Care. Health Policy. Health Services. Public Health.

\title{
INTRODUÇÃO
}

$\mathbf{0}$

envelhecimento populacional e a transição epidemiológica, com aumento das doenças crônicas, são condições emergentes na população brasileira. Frequentemente as doenças crônicas são combinadas, associadas a outras comorbidades ou agravadas pelas alterações próprias do envelhecimento, o que limita a possibilidade de tratamentos curativos e impõe a demanda de uma atenção integral para atender as necessidades físicas, psicossociais e espirituais dos indivíduos acometidos, ainda que nem sempre alcance a cura da doença. Este contexto, associado à escassez de recursos aplicados à saúde, cria um desafio para a efetividade das políticas de saúde, bem como exige maior preparo dos profissionais envolvidos ${ }^{12}$.

O processo prolongado e gradativo das doenças crônicas implica num longo enfrentamento, tanto pelo paciente quanto para quem cuida deste (familiares e profissionais de saúde), e envolve decisões sobre as mudanças de rotina, manejo de gastos com saúde e conflitos sobre o processo de final de vida. Além disso, estas condições têm um impacto importante sobre o sistema de saúde, como o aumento da demanda sobre os serviços de saúde, a necessidade de atenção longitudinal e envolvimento de diversos profissionais na trajetória da doença e no planejamento do cuidado. O último ano de vida também tem sido associado ao período de maior demanda de cuidados e gasto com tratamentos de saúde?.

Para lidar com a complexidade das doenças que ameaçam a vida, e que incluem as doenças crônicas não transmissiveis, doenças em fase avançada, as condições de fragilidade e outras sem disponibilidade de 
tratamento curativo, os cuidados paliativos (CP) passam a ser uma resposta possível no sistema de saúde, amplamente recomendada, para oferecer suporte para esses problemas emergentes, com o objetivo principal de melhorar a qualidade de vida, respeitar a autonomia, prevenir o sofrimento e controlar os sintomas, integrando a atenção dos aspectos físicos, psicológicos, sociais, familiares e existenciais, de forma a ser aplicado desde o diagnóstico de uma doença que ameace a vida, até sua aplicação, mais abrangente, no fim de vida e no luto 13.

Inerentemente ao seu conceito e às suas propostas, a forma de atuação dos CP vai ao encontro da proposta e do conceito da clínica ampliada do Sistema Único de Saúde (SUS), defendida por alguns pesquisadores $^{4}$ e, nos últimos anos, fortaleceu-se a recomendação da aplicação dos CP nos sistemas de saúde com o importante marco, em 2014, da publicação da Resolução nº 19 da Organização Mundial da Saúde (OMS), na $67^{a}$ Assembleia Mundial de Saúde, específica para o fortalecimento dos CP na atenção à saúde ${ }^{5}$. A partir desta, houve avanços na legislação brasileira, tanto de abrangência nacional como nas esferas estaduais, para a sua difusão no SUS. Em 2018, foi aprovada a Resolução da Comissão Intergestores Tripartite (CIT) n 41, de 31 de outubro de 2018, que dispõe sobre as diretrizes para a organização dos cuidados paliativos no âmbito do SUS6. No Paraná aprovou-se a Lei Estadual n² 20.091, de 19 de dezembro de 2019, sobre a instituição dos preceitos e fundamentos dos Cuidados Paliativos no estado7. No entanto, seu desenvolvimento ainda se encontra na fase inicial, com diversas barreiras para a sua disseminação, falta de diretrizes para a sua organização e aplicação nas Redes de Atenção à Saúde (RAS) e nos serviços de saúde.

Assim, este estudo busca identificar a demanda atual de CP no estado do Paraná, em particular para o SUS, revisar a legislação pertinente ao tema e apresentar os fundamentos desta forma de abordagem. reflexões sobre a necessidade de inserir os CP nos serviços de saúde e na organização das Redes de Atenção à Saúde (RAS), e propor ações para direcionar e disseminar sua aplicação no sistema de saúde do estado.

\section{MÉTODO}

Realizou-se a análise dos dados de mortalidade do estado do Paraná, em 2018, pelo Sistema de Informações de Mortalidade (SIM/DATASUS) ${ }^{9}$. Foi utilizada estatística descritiva para identificar os aspectos de mortalidade relacionados à: a) faixa etária; b) demanda populacional de CP no fim de vida, por meio das causas de morte associadas pela Classificação Internacional de Doenças (CID-10), conforme método proposto na literatura ${ }^{10}$; e a frequência dos principais locais de ocorrência de mortes. Nestes dados foram analisadas as diferenças por Regionais de Saúde do estado do Paraná, conforme o banco de dados público.

Também se realizou a busca, nos canais oficiais das entidades públicas, nas legislações federal e estadual vigentes relacionadas à temática dos CP. para identificar o atual contexto legislativo e sua abrangência para a inserção dos CP nas políticas públicas de saúde do Paraná.

\section{RESULTADOS}

Os estados do Sul do Brasil têm maiores percentuais de indivíduos idosos na população. Atualmente, a expectativa de vida no Brasil, em 2019, foi estimada em 76,5 anos e, espera-se um aumento até próximo de 81 anos, até 2060. Já a proporção de idosos acima de 65 anos, saltará dos atuais 9,5\% para até 25,5\%, em 
2060. O Paraná, que já tem aproximadamente 10,3\% de idosos, ultrapassará os 20\% em 2040 e terá 27\% em $2060^{8}$.

O aumento da população idosa associa-se à ocorrência de morte em faixas etárias mais avançadas, como consequência da maior prevalência das doenças crônicas. Em 2018, verificou-se que, das 73.719 mortes ocorridas no Paraná, aproximadamente 70\% eram de pessoas com mais de 60 anos de idade (Figura 1). sendo que a Regional de Saúde (RS) com maior porcentagem de mortes acima de 60 anos foi a $22^{a}$ RS (Sede Ivaiporã), com 74,6\% e a com menor proporção foi a $1^{\text {a }}$ RS (Paranaguá), com 63,7\%.

Figura 1 - Frequência de mortes por faixa etária no Paraná, em 2018.

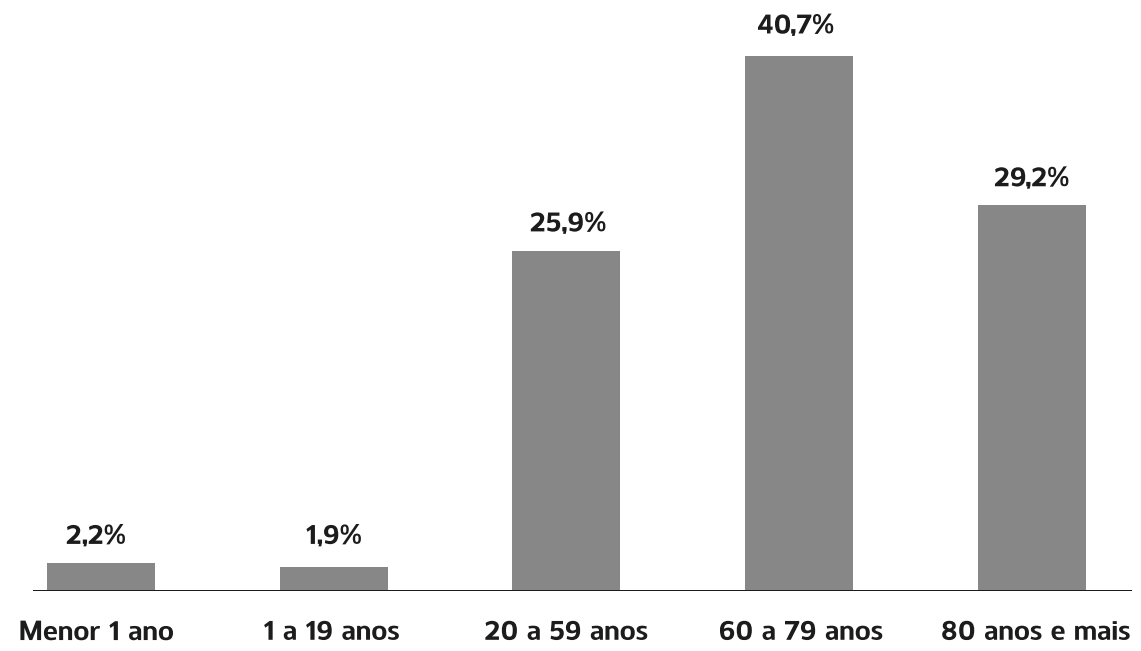

Fonte: Brasil, $2020^{9}$

Numa projeção populacional, considerando as causas de morte e o método de estimativa populacional de CP10, observou-se que 63,8\% das mortes ocorridas no Paraná, ou seja, 47.000 mortes em 2018, tinham potencial necessidade de CP no fim de vida. As regionais de saúde com maior porcentagem de mortes com potencial necessidade de CP foram a $8^{a}$ RS (Francisco Beltrão) com 69,5\% e a $4^{a}$ RS (Irati) com 68,7\%, enquanto a com menor frequência foi a $1^{\text {a }} \mathrm{RS}$ (Paranaguá) com 56,5\%, possivelmente em decorrência do maior número de morte em faixas etárias mais jovens ${ }^{9}$.

Em estudo realizado com diversos paises, projeta-se que estas condições tendem a aumentar nas próximas décadas, em particular para as doenças crônicas associadas à obesidade, síndromes demenciais e câncer, e, até 2040, podem chegar próximo de $80 \%$ de todas as mortes no mundo, sendo a maioria nos países de baixa e média renda"1.

Dentre estas mortes mais comumente associadas à necessidade de CP, as doenças cardiovasculares, cerebrovasculares, oncológicas e respiratórias são as mais frequentes e, juntas, correspondem por 90\% das demandas por atenção em CP (Figura 2) ${ }^{9}$. 
Figura 2 - Distribuição das causas de morte com potencial necessidade de cuidados paliativos no fim de vida no Paraná, em 2018.

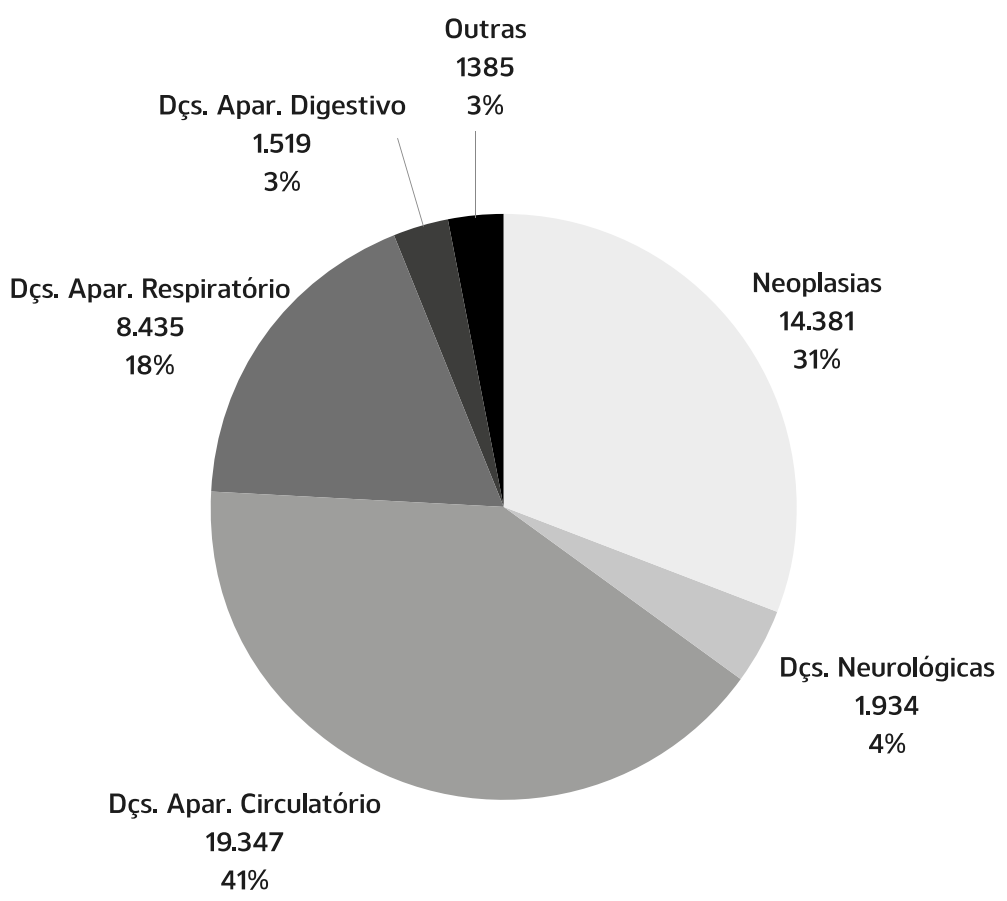

Fonte: Brasil, $2020^{9}$

No Paraná, 66,6\% das mortes ocorrem nos hospitais, 21,3\% no domicílio, 4,9\% em outros estabelecimentos de saúde (unidades de pronto atendimento, unidades básicas de saúde, consultórios, etc.), 3,8\% em vias públicas e 3,3\% em outros locais. No entanto, observa-se uma variação nesta distribuição no estado. Enquanto na $17^{a}$ RS (Londrina) 78\% das mortes ocorrem nos hospitais e 14,9\% no domicílio, na $21^{\text {a }}$ RS (Telêmaco Borba) 54,6\%

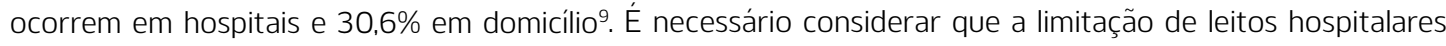
numa localidade pode ocasionar a transferência do paciente para outras regionais, e a morte em domicilio, se não houver disponibilidade de atenção domiciliar, pode ser associada à limitação de acesso a serviços de saúde ou de assistência profissional².

\section{DISCUSSÃO}

Há uma perspectiva crescente da proporção da população idosa no estado do Paraná para as próximas décadas, o que gerará aumento da demanda de assistência a esta população. Atualmente, 70\% das mortes ocorrem em pessoas com mais de 60 anos, com variação entre as regionais de saúde do estado entre 75\% e 64\%. Esta proporção é maior que a média brasileira, em torno de 63 a 65\%².

Do total de mortes ocorridas no estado, cerca de 64\% têm potencial necessidade de suporte em CP no fim de vida, ou seja, estão associadas a doenças crônicas ou comorbidades que afetam a qualidade de vida, em geral por longos períodos, principalmente pelas doenças cardiovasculares, cerebrovasculares, oncológicas 
e respiratórias, que demandam cuidados contínuos e assistência frequente. Tais dados são condizentes com as principais causas de morte no Brasil, mas em proporções maiores que a média brasileira². A variação entre as regionais de saúde foi de $69 \%$ para a $8^{\mathrm{a}} \mathrm{RS}$ (Francisco Beltrão) e a $4^{\mathrm{a}} \mathrm{RS}$ (Irati), com menor frequência para a $1^{\mathrm{a}} \mathrm{RS}$ (Paranaguá), com 56\%.

No Paraná, a maioria das mortes ocorrem nos hospitais (67\%) e 21\% em domicílios. A regional com maior proporção de óbito hospitalar foi a $17^{a}$ RS (Londrina) (78\%) e a com menor proporção foi a $21^{a}$ RS (Telêmaco Borba), com 55\%. A média nacional também é de aproximadamente 67\%, com os estados do Sul, Sudeste e Centro-Oeste com maior proporção, entre 67 e 72\%, e os do Norte e Nordeste entre 56 e 60\%².

Deve-se considerar que o sistema público de saúde brasileiro não oferece outras opções fora dos ambientes hospitalares para a assistência de pessoas com condições limitantes de vida, como os hospices (serviços exclusivos de cuidados paliativos), unidades de cuidados continuados integrados, instituições de longa permanência (ILP) ou atenção domiciliar, de forma ampla, quando comparado com outros países. Assim, uma maior proporção de óbitos em casa pode indicar falta de estrutura de saúde ou de acesso ao suporte adequado, nas condições com risco de vida².

\section{CUIDADOS PALIATIVOS NA REDE DE ATENÇÃO Ȧ SAÚDE}

O marco inicial dos CP contemporâneos é creditado à abertura do Saint Christopher's Hospice, no Reino Unido, em 1967, por Dame Cecily Saunders, que, inicialmente, atuava como enfermeira no cuidado de doentes terminais, principalmente de câncer, e, depois, graduou-se em administração pública e social. Em 1957, formou-se em medicina e, percebendo a necessidade de melhor controle dos sintomas, como a dor e o sofrimento, principalmente dos pacientes com câncer em fase avançada, elaborou diretrizes para um cuidado mais holístico e especializado destes pacientes e de suas famílias ${ }^{112}$.

Um dos pontos mais importantes de sua fundamentação teórica e prática foi o conceito de "Dor Total", que busca o controle aprimorado do sintoma da dor física, além da ampla consideração dos aspectos psicossociais, familiares e existenciais, que afetam diretamente no sofrimento vivido pelo paciente. A partir da década de 80, a OMS iniciou a divulgação de políticas de controle da dor e atenção oncológica, e adotou o termo "Palliative Care" nos documentos oficiais, utilizando-se do termo empregado pelo cirurgião oncologista Balfour Mount, do Canadá, para descrever a aplicação desta abordagem no Royal Victoria Hospital, já que o termo hospice, na língua francesa, tinha um tom pejorativo e associado aos asilos ou hospedagens de baixa qualidade ${ }^{1,13}$.

Os CP têm como principais fundamentos ${ }^{1,3,7}$ :

- A prevenção, avaliação e controle apurado dos sintomas físicos, psíquicos, sociais e espirituais, com atuação no suporte das questões relacionadas ao fim da vida;

- Afirmar a vida com qualidade e autonomia, aceitando a morte como parte desta, sem prolongar ou antecipar sua ocorrência, ou seja, evitando a distanásia/mistanásia e a eutanásia;

- Evitar a realização de medidas terapêuticas obstinadas ou exageradas, tanto aquelas para prolongar extensamente o tempo de vida sem qualidade quanto as intervenções que não trazem benefícios claros para a qualidade de vida do paciente, ainda que a doença não seja possível de ser curada;

- Oferecer suporte multiprofissional para permitir o alcance dos diversos problemas relacionados às doenças e ao momento vivido, incluídos de forma mais precoce possível, mesmo que associado aos 
tratamentos com enfoque curativo (modificadores da doença);

- Oferecer comunicação compreensiva e escuta ativa para o paciente e familiares, abordando aspectos que vão além daqueles específicos da doença, como as questões sociais, familiares e psicológicas;

- $\quad$ Estender o olhar e incluir o suporte para os familiares e cuidadores, durante a trajetória de vida do paciente e na assistência do luto:

- $\quad$ Permitir o exercício da autonomia e independência, tanto quanto possível.

No Brasil, apesar de ações isoladas anteriores, considera-se a inauguração da Unidade IV do Instituto Nacional do Câncer (INCA), em 1998, como um marco na aplicação dos CP no SUS³. Em 2002, o Ministério da Saúde publicou a Portaria n 19/2002, que estabeleceu o Programa Nacional de Assistência à Dor e Cuidados Paliativos, voltado principalmente para ampliar o acesso a medicações de controle de dor, mas de alcance limitado à sintomatologia física, sem abordar integralmente outros aspectos específicos dos cuidados paliativos, como o suporte familiar, o trabalho interdisciplinar, o controle de outros sintomas psicológicos e físicos, a atuação profissional da morte e do morrer e o cuidado com o luto' ${ }^{14}$

Segundo a Organização Mundial da Saúde, para a integração dos CP no sistema de saúde é necessário desenvolver os seguintes elementos chaves: 1) Estabelecer políticas públicas específicas para o seu desenvolvimento; 2) Disponibilidade de medicação para o controle de sintomas, particularmente o acesso aos opioides; 3) Educação dos profissionais de saúde e da população em geral para sua disseminação e compreensão e; 4) Implementar nos serviços de saúde a abordagem dos CP. em todos os níveis de atenção. Para todos estes elementos deve haver metas de curto, médio e longo prazos, e indicadores mensuráveis ao longo do tempo. A sua difusão deve considerar o contexto cultural e socioeconômico, as demandas epidemiológicas e demográficas, e as particularidades do sistema de saúde do local ou do País ${ }^{1,5}$.

Mais recentemente, um marco importante foi estabelecido pela Resolução nº 19 da OMS, em 2014 que recomenda a inserção dos CP em todos os níveis de atenção à saúde ${ }^{5}$. No Brasil, destaca-se a recente Resolução CIT n 41/2018', com diretrizes para a organização dos cuidados paliativos, associado aos cuidados continuados integrados. E no contexto do estado do Paraná, a recomendação foi fortalecida pela Lei Estadual n² 20.091/20197. Dentre os diversos avanços presentes na Lei Estadual, estabelece-se que para a organização dos cuidados paliativos, deve-se:

I - integrar os cuidados paliativos em todos os níveis da Rede de Atenção à Saúde:

II - promover a melhoria da qualidade de vida das pessoas enfermas por doenças extensas e potencialmente fatais;

III - incentivar o trabalho em equipe multiprofissional, sendo esta constituída minimamente por profissionais de medicina, enfermagem, serviço social, psicologia e, quando possível e conforme necessidade, por profissionais nutricionista, terapeuta ocupacional, fisioterapeuta, farmacêutico, odontólogo, assistente espiritual e fonoaudiólogo;

IV - fomentar a inclusão de conteúdos disciplinares sobre cuidados paliativos nos cursos técnicos, de graduação e de pós-graduação da área da saúde;

V - ofertar educação permanente em cuidados paliativos para os profissionais que já atuam na assistência com ações paliativas; 
VI - promover a disseminação de informações sobre cuidados paliativos na sociedade:

VII - ofertar medicamentos que promovam o controle dos sintomas das pessoas enfermas.

Qualquer profissional de saúde pode adotar abordagens e ações paliativas, incluindo profissionais generalistas e da atenção primária, a partir de um conhecimento básico para oferecer suporte aos pacientes com necessidades específicas e conforme sua complexidade clínica' (Figura 3).

Figura 3 - Distribuição populacional no acesso a serviços de Cuidados Paliativos, por complexidade.

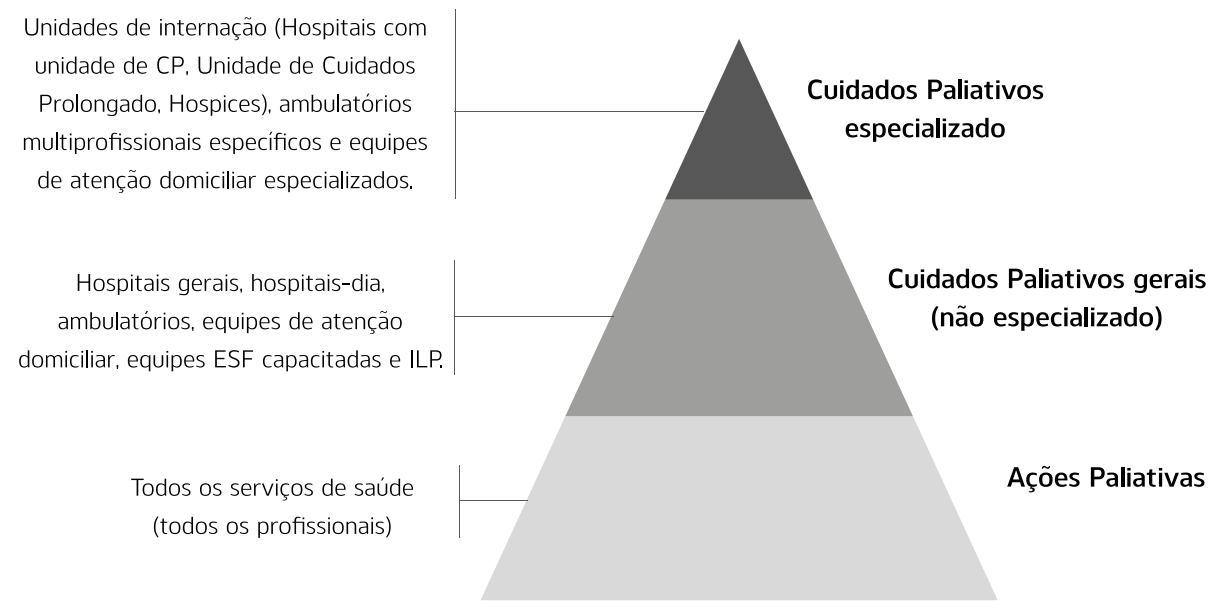

Fonte: Elaborado pelos autores, 2020.

As Redes de Atenção à Saúde (RAS) são as principais formas de organização dos serviços do SUS e visam orientar a integração dos serviços de saúde existentes, nos diversos níveis de atenção, de forma a atuar positivamente nos resultados a serem buscados no campo da saúde. As redes de saúde têm a Atenção Primária à Saúde (APS) como coordenadora do cuidado e ordenadora das ações, para superar a fragmentação da continuidade da assistência oferecida. O objetivo principal das RAS é a integração sistêmica, de ações e serviços de saúde, para prover o cuidado continuado, integral, acessível e equitativo, com qualidade e eficiência clínica e financeira, responsável e humanizado ${ }^{15}$.

São atributos essenciais ao funcionamento das RAS a "extensa gama de estabelecimentos de saúde que prestam serviços de promoção, prevenção, diagnóstico, tratamento, gestão de casos, reabilitação e cuidados paliativos e integram os programas focalizados em doenças, riscos e populações específicas, os serviços de saúde individuais e os coletivos"15. A aplicação de CP não deve ser limitada a serviços especializados, podendo ser aplicados em todos os níveis de atenção, ou seja, pode ser aplicado onde quer que o paciente esteja, no domicílio, nos serviços de saúde da comunidade, hospitais gerais, ambulatórios, hospitais-dia, instituições de longa-permanência (ILP), entre outros (Figura 4). 
Figura 4 - Organização das Redes de Atenção à Saúde para o suporte de pacientes com necessidade de cuidados paliativos

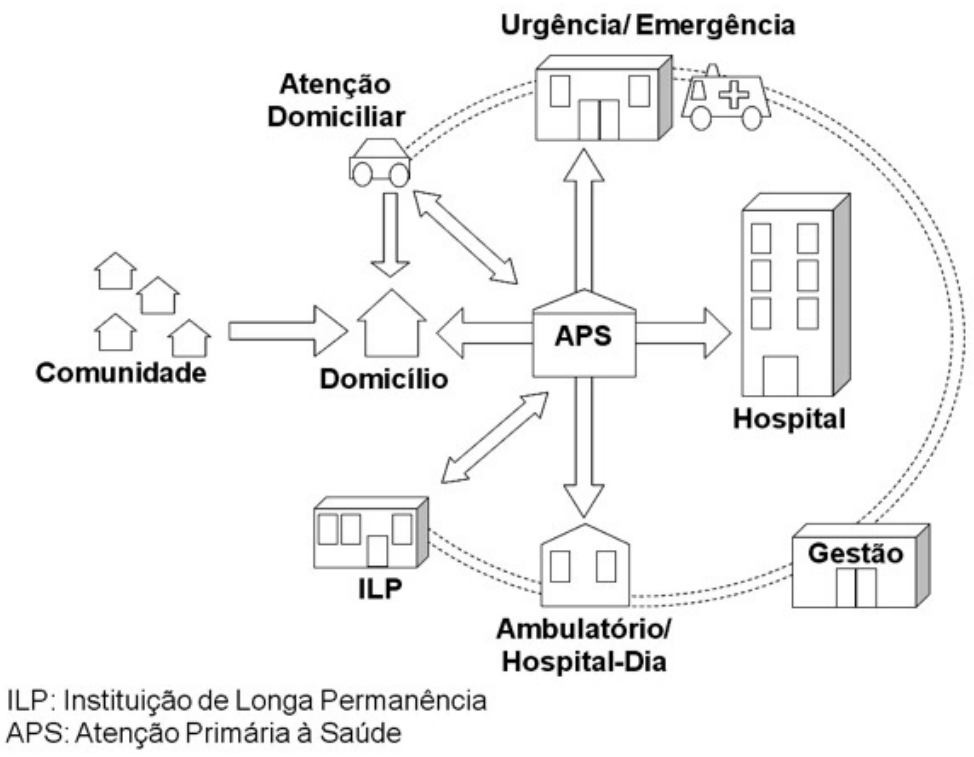

Fonte: Elaborado pelos autores, 2020

O estado do Paraná tem sido palco de diversas iniciativas pioneiras para a aplicação do CP no sistema público de saúde, com destaque para: a criação e funcionamento do Grupo Interdisciplinar de Suporte Terapêutico Oncológico (GISTO) do Hospital Erasto Gaertner, em Curitiba, um dos primeiros serviços de suporte de pacientes oncológicos fora das possibilidades curativas, iniciado em 1994 e que, em 2020, inaugurou sua unidade hospice; formação da primeira equipe de internação domiciliar exclusiva para pacientes em CP, em 2002, pela prefeitura de Londrina; a formação da Equipe Interdisciplinar de Cuidados Paliativos Oncológicos (EICPO) do Hospital de Câncer de Londrina, desde 2006, e que, em 2016, abriu uma ala de internação exclusiva; além de iniciativas de disponibilização de leitos de internação exclusiva para pacientes em CP nos hospitais estaduais Zona Norte e Zona Sul de Londrina, entre outras em diferentes regiões do estado.

No entanto, a prática dos CP ainda não é institucionalizada no sistema de saúde, e é pouco difundida nas RAS, com aquelas na Rede de Urgência e Emergência, na Rede de Atenção ao Idoso e aquelas relacionadas ao controle das doenças crônicas. Assim, há limitação para a continuidade desta abordagem entre os diferentes serviços ou níveis de atenção.

\section{AÇÕES NECESSÁRIAS PARA A APLICAÇÃO DOS CUIDADOS PALIATIVOS NOS DIFERENTES NIVEIS DE ATENÇÃO}

Dentre as ações transversais, necessárias a todos os níveis de atenção à saúde, citam-se': 
- Capacitar as equipes de saúde dos diferentes serviços para oferecer ações paliativas, incluindo o controle efetivo da dor e outros sintomas físicos, psíquicos e sociais frequentemente encontrados nos pacientes com doenças que ameaçam a vida ou condições crônicas que causam sofrimento e afetam a qualidade e continuidade da vida, incluindo o planejamento do cuidado e suporte de fim de vida:

- Sistematizar a identificação dos pacientes com indicação de CP pelos serviços de saúde, conforme o nível de complexidade e contexto de atuação;

- Organizar a oferta e o acesso aos serviços de CP, compartilhamento de informações dos pacientes e o fluxo entre os serviços, para a continuidade do cuidado;

- Disseminação e aceitação pelos profissionais pela decisão compartilhada, incluindo a recusa de tratamentos desconfortáveis ou invasivos sem retorno proporcional de sobrevida, respeito dos aspectos éticos e da autonomia dos individuos diretamente afetados, incluindo o conhecimento sobre documentos específicos, como as Diretrizes Antecipadas de Vontade (DAV);

- Fomentar e otimizar o uso dos recursos públicos utilizados na assistência à saúde, particularmente na Média e Alta Complexidade, reduzindo a hospitalização e demanda de vagas de maior complexidade, por meio da atuação preventiva, uso racional das tecnologias e apropriação dos aspectos relacionados à humanização da assistência.

\section{ATENÇÃO PRIMÁRIA}

Tanto a Atenção Primária à Saúde quanto os Cuidados Paliativos pressupõem o cuidado integral ao indivíduo, ampliação da participação social e familiar, oferecem orientação, ações preventivas, tratamento e cuidados no contexto de vida do paciente, mesmo nos casos em que não há possibilidade de cura. A Atenção Básica tem diversas vantagens na oferta de ações paliativas, como a maior proximidade do paciente e seus familiares e o envolvimento por períodos maiores, o que favorece a confiança e conhecimento pelos profissionais, e devem ser ordenadoras dos cuidados nas RAS 1.16

Recentemente os CP foram incluídos na Carta de Serviços da Atenção Básica. Entre as ações a serem realizadas estão: a identificação sistematizada dos pacientes com necessidade de cuidados paliativos por meio de ferramentas avaliativas próprias ou validadas, associada à avaliação dos profissionais de saúde capacitados; o acompanhamento e a oferta de ações para prevenção de complicações decorrentes da progressão da doença, controle adequado dos sintomas físicos, psíquicos e sociais, com suporte de equipes multiprofissionais; suporte e orientação aos cuidadores informais e familiares para promover o planejamento dos cuidados até as fases mais avançadas da doença, com promoção da autonomia e individualidade das decisões; incluir o suporte do óbito no domicílio com assistência adequada e no luto dos familiares, por meio da identificação de necessidades psicossociais, com encaminhamento para o suporte adequado, quando necessário ${ }^{117}$.

As equipes multiprofissionais são essenciais na oferta de CP na RAS, e podem ser ofertados pelos Núcleos Multiprofissionais de Apoio à Saúde da Família, pelos Serviço de Atenção Domiciliar e ambulatórios de referências. $O$ apoio multidisciplinar busca ampliar o suporte para o controle adequado dos sintomas físicos, psíquicos e sociais, por meio de matriciamento ou atuação direta com o paciente e familiares, incluindo a possibilidade de óbito no domicílio com assistência adequada, com controle dos sintomas e oferta de medicamentos analgésicos de maior efetividade (opióides) ${ }^{118}$. 


\section{SERVIÇOS DE URGÊNCIA/EMERGÊNCIA}

A adoção de ações em CP na Rede de Urgência e Emergência é fundamental para a organização do fluxo dos pacientes entre os serviços de saúde. Frequentemente pacientes com doenças crônicas em fase avançada são atendidos em Unidade de Pronto Atendimento (UPA). Pronto-Socorros e pelo Serviço de Atendimento Médico de Urgência (SAMU). Assim, há necessidade de: estabelecer a rotina de identificação de pacientes com potencial necessidade de CP e adotar ferramentas para reconhecimento daqueles já acompanhados por serviços de referência em CP; oferecer medidas de alivio de sofrimento para as fases finais de vida, incluindo a possibilidade de óbito sem adoção de medida de prolongamento artificial de vida, quando há consenso adequado entre profissionais, paciente e familiares ou cuidadores responsáveis; e estabelecer na rotina de comunicação a sinalização de pacientes com indicação de CP quando realizar a transferência para um serviço de saúde!.

\section{MÉDIA E ALTA COMPLEXIDADE}

Ao longo da trajetória de vida, os pacientes transitam pelos diferentes níveis de atenção à saúde. Na média complexidade, pacientes com necessidade de CP têm necessidade de acessar ambulatórios de especialidades, sendo necessário sensibilizar para esta abordagem os serviços ambulatoriais, como os de geriatria, pneumologia, cardiologia, neurologia, oncologia, etc., bem como considerar a possibilidade de serviços ambulatoriais de referência em CP!

Estudos indicam que no último ano de vida do paciente há uma alta frequência de reinternações hospitalares, e ainda deve-se considerar que a maioria das mortes, mesmo por doenças crônicas, ocorrem em hospitais ${ }^{19}$. Estas constantes reinternações indicam a necessidade de um sistema de comunicação eficiente entre os serviços de saúde (nos diferentes níveis de atenção) e adequação do sistema de referência e contrarreferência para manter a continuidade do cuidado na trajetória da doença pois, quando não há integração, há a possibilidade de cada admissão hospitalar ser considerada como um evento agudo e tratada de forma curativa, com repetição de exames diagnósticos e adoção de medidas intensivas e invasivas nos setores de emergência18.

Nos hospitais, a inserção de CP possibilita a percepção de um melhor atendimento dos pacientes com doenças em fase avançada e seus familiares, além de otimizar a alocação de recursos, evitando procedimentos ou tratamentos excessivos, reduzir a demanda por leitos de unidade de tratamento intensivo (UTI), promover um melhor controle dos sintomas desconfortantes e promover um maior tempo de acompanhamento familiar ${ }^{12}$.

Para estes serviços, a constituição de comissões internas ou equipes de referência em CP podem favorecer a aplicação desta abordagem e devem ser compostas minimamente de um médico, um enfermeiro e um profissional de serviço social ou psicólogo, sendo ideal a participação de outras profissões da área da saúde. Os serviços hospitalares devem identificar os pacientes com necessidade de cuidados paliativos na sua rotina e otimizar as ações de prevenção, avaliação e controle adequado dos sintomas físicos, psíquicos e sociofamiliares, incluindo o planejamento dos cuidados nas fases avançadas das doenças, promover a autonomia, a decisão compartilhada e a individualidade nas opções do suporte prestado, e oferecer as medidas de suporte para a ocorrência da morte sem adoção de medida de prolongamento artificial de vida e com controle adequado dos sintomas ${ }^{2,3}$.

Como opção intermediária à internação devem ser consideradas a instituição de hospitais-dia, 
Unidades de Cuidados Prolongados (UCP) ou instituições de longa permanência (ILP), para incluir na sua oferta de serviços a adoção da abordagem multiprofissional dos CP. ou mesmo ter serviços de referência especializados (hospices) para a oferta de estadia do paciente e apoio aos familiares ${ }^{1.3}$.

\section{IMPLICAÇÃO PARA OS RECURSOS DISPONIVEIS DO SISTEMA DE SAÚDE}

Além da busca pela qualidade de vida, estima-se que com o acesso dos pacientes aos CP há uma redução de custos no atendimento oferecido, em comparação ao tratamento exclusivamente curativo. Primeiramente, porque os CP são considerados uma atenção de menor complexidade, baixo custo e menor demanda tecnológica, visto que evita tratamentos excessivos que não trazem benefício ao paciente, e que são geralmente os mais onerosos para o sistema de saúde, como a repetição de exames diagnósticos, procedimentos invasivos e diagnósticos, e reduzem a demanda por leitos de UTR2.

As ações paliativas focam na prevenção de complicações e utilização de recursos menos invasivos, incentivam a autonomia do paciente na escolha dos tratamentos oferecidos e visam oferecer assistência adequada para o controle de sintomas no ambiente domiciliar ou mesmo oferecer suporte para a ocorrência do óbito no domicílio, o que possibilita que o indivíduo viva o processo de morte no seu ambiente familiar. quando esse é possível ${ }^{3}$.

Para a gestão dos serviços de saúde, a disponibilidade de CP pode ser atraente ao considerar os aspectos econômicos. Diversos estudos indicam que a inclusão dos CP próximos à comunidade, como nos serviços de atenção primária, tem sido associada a um melhor controle de sintomas, melhora na satisfação dos pacientes, menores taxas de internações e reinternações hospitalares e menor custo que os tratamentos usuais. Também foi associado a um aumento da chance de óbito em domicílio e maior tempo em casa no último ano de vida, em comparação com o tratamento usual21.

\section{CONCLUSÃO}

A demanda crescente de suporte e assistência aos pacientes com doenças crônicas e ao envelhecimento populacional, em particular no estado do Paraná, trazem problemas emergentes que poderão ser abordados pela prática dos CP. considerando as particularidades das regionais de saúde e dos serviços integrados às Redes de Atenção à Saúde, otimizando o uso dos recursos do sistema de saúde e buscando oferecer a estes pacientes uma melhor qualidade do cuidado, mais autonomia e preparo para lidar com os desafios destas condições, até o fim da vida.

Recentemente houve importantes avanços para pavimentar a inserção dos CP na assistência à saúde, tanto na legislação federal quanto estadual, e nas iniciativas presentes nos serviços de saúde. Em particular no Paraná, destaca-se o pioneirismo na oferta desta forma de abordagem no sistema público e a definição de uma lei estadual para fomentar sua aplicação. No entanto, a área ainda se encontra na fase de desenvolvimento e difusão, e necessita integrar-se às Redes de Atenção à Saúde e serem inseridos nos serviços de saúde.

Para ofertar e ampliar o acesso dos pacientes com doenças que ameaçam a vida e condições que afetam a qualidade desta, são necessárias ações de CP na atenção primária, nos serviços de urgência e emergência, na média e alta complexidade. Também é importante fomentar a capacitação profissional e incluir a temática dos cuidados paliativos no currículo de graduação e residências da área da saúde. 


\section{REFERÊNCIAS}

1. Gómez-Batiste XC, Stephen. Building Integrated Palliative Care Programs and Services. Catalonia (Espanha): Liberdúplex; 2017.

2. Marcucci FCI CM, Rosenberg JP, Yates P. Tendências nos locais de óbito no Brasil e análise dos fatores associados em idosos de 2002 a 2013. Geriatr Gerontol Aging. 2017:11(1):10-7.

3. ANCP. Academia Nacional de Cuidados Paliativos. Manual de cuidados paliativos. 2. ed. Rio de Janeiro: Diagraphic; 2012.320 p.

4. Campos GWdS, Amaral MAd. A clínica ampliada e compartilhada, a gestão democrática e redes de atenção como referenciais teórico-operacionais para a reforma do hospital. Ciência \& Saúde Coletiva. 2007:12:849-59. Disponível em: http://www.scielo.br/scielo.php?script=sci_ arttext\&pid=S1413-81232007000400007\&nrm=iso.

5. WHO. World Health Organization. 67 $7^{\mathrm{a}}$ World Health Assembly Resolution 67.19 - Strengthening of Palliative Care as a Component of Comprehensive Care Throughout the Life Course. Genebra: WHO; 2014

6. Brasil. Ministério da Saúde/Comissão Intergestores Tripartite (CIT). Resolução N 41, de 31 de outubro de 2018. Dispõe sobre as diretrizes para a organização dos cuidados paliativos, à luz dos cuidados continuados integrados, no âmbito Sistema Único de Saúde (SUS). 2018.

7. Paraná. Lei 20.091, de 19 de Dezembro de 2019, dispõe sobre a instituição dos preceitos e fundamentos dos Cuidados Paliativos no Paraná: Curitiba; 2019

8. IBGE. Instituto Brasileiro de Geografia e Estatística. População - Projeção da população do Brasil e das Unidades da Federação. Disponível em: https://www.ibge.gov.br/apps/populacao/projecao/.

9. Brasil. Ministério da Saúde. DATASUS - Informações de Saúde (TABNET). Estatísticas Vitais - Mortalidade geral. Paraná - Dados preliminares 2018. Brasília: 2020. Disponivel em: http://tabnet.datasus.gov.br/cgi/deftohtm.exe?sim/cnv/pobt10pr.def:

10. Murtagh FE, Bausewein C, Verne J, Groeneveld El, Kaloki YE, Higginson IJ. How many people need palliative care? A study developing and comparing methods for population-based estimates. Palliative Medicine. 2013;28(1):49-58. Disponível em: http://pmj.sagepub.com/ content/early/2013/05/20/0269216313489367

11. Etkind SN, Bone AE, Gomes B, Lovell N, Evans CJ, Higginson IJ, et al. How many people will need palliative care in 2040 ? Past trends, future projections and implications for services. BMC Med. 2017:15(1):102. PubMed PMID: 28514961. eng. Disponivel em: https://www.ncbi. nlm.nih.gov/pubmed/28514961

12. Institute CS. Dame Cicely Saunders Biography. Disponível em: http://cicelysaundersinternational.org/dame-cicely-saunders/.

13. Phillips D. McGill University. Palliative Care McGill - Portraits: Balfour Mount. Disponivel em: https://www.mcgill.ca/palliativecare/portraits-0/balfour-mount

14. Brasil. Ministério da Saúde. Portaria n 19/GM de 03 de janeiro de 2002. Institui, no âmbito do Sistema Único de Saúde, o Programa Nacional de Assistência à Dor e Cuidados Paliativos: Brasília; 2002.

15. Brasil. Ministério da Saúde. Portaria MS n 4.279. Estabelece diretrizes para a organização da Rede de Atenção à Saúde no âmbito do Sistema Único de Saúde (SUS): Brasília; 2010. Disponivel em: http://bvsms.saude.gov.br/bvs/saudelegis/gm/2010/prt4279_30_12_2010.html:

16. Marcucci FCl, Cabrera MAS, Perilla AB, Brun MM, de Barros EML, Martins VM, et al. Identification and characteristics of patients with palliative care needs in Brazilian primary care. BMC Palliat Care. 2016;15(1):1-10. Disponível em: http://dx.doi.org/10.1186/s12904-016-0125-4.

17. Brasil. Ministério da Saúde. Carteira de Serviços da Atenção Primária à Saúde (CaSAPS). Brasília: Ministério da Saúde; 2019.

18. Marcucci F. Identificação e caracterização dos indivíduos com indicação de Cuidados Paliativos cadastrados na Estratégia Saúde da Família [Tese de Doutorado] 121 f.: il. Londrina: Universidade Estadual de Londrina; 2016.

19. NEoLCIN: National End of Life Care Intelligence Network. What do we know now that we didn't know a year ago?. 2012: Disponível em: http://www.endoflifecare-intelligence.org.uk/view?rid=464:

20. Pham B, Krahn M. End-of-life care interventions: an economic analysis. Ont Health Technol Assess Ser. 2014;14(18):1-70.

21. Samantha S, Aoife B, Sinéad OH, Charles N. Evidence on the cost and cost-effectiveness of palliative care: A literature review. Palliative Medicine. 2013 2014/02/01;28(2):130-50. Disponivel em: https://doi.org/10.1177/0269216313493466. 